KYUNGPOOK Math. J. 53(2013), 191-205

http://dx.doi.org/10.5666/KMJ.2013.53.2.191

\title{
Meromorphic Functions Sharing a Nonzero Polynomial IM
}

PulaK SAHOO

Department of Mathematics, University of Kalyani, West Bengal-741235, India.

e-mail : sahoopulak@yahoo.com; sahoopulak1@gmail.com

ABSTRACT. We study the uniqueness of meromorphic functions concerning nonlinear differential polynomials sharing a nonzero polynomial IM. Though the main concern of the paper is to improve a recent result of the present author [12], as a consequence of the main result we also generalize two recent results of X. M. Li and L. Gao [11].

\section{Introduction, Definitions and Results}

In this paper, by meromorphic functions we will always mean meromorphic functions in the complex plane. We adopt the standard notations in the Nevanlinna theory of meromorphic functions as explained in [7], [15] and [16]. For a nonconstant meromorphic function $h$, we denote by $T(r, h)$ the Nevanlinna characteristic of $h$ and by $S(r, h)$ any quantity satisfying $S(r, h)=o\{T(r, h)\}$ as $r \rightarrow \infty$ possibly outside a set of finite linear measure. A meromorphic function $a(z)(\not \equiv \infty)$ is called a small function with respect to $f$, provided that $T(r, a)=S(r, f)$.

Let $f$ and $g$ be two nonconstant meromorphic functions, and let $a$ be a finite value. We say that $f$ and $g$ share the value $a \mathrm{CM}$ (counting multiplicities), provided that $f-a$ and $g-a$ have the same set of zeros with the same multiplicities. Similarly, we say that $f$ and $g$ share $a$ IM (ignoring multiplicities), provided that $f-a$ and $g-a$ have the same set of zeros ignoring multiplicities.

In 1959, W. K. Hayman (see [6], Corollary of Theorem 9) proved the following theorem:

Theorem A. Let $f$ be a transcendental meromorphic function and $n(\geq 3)$ is an integer. Then $f^{n} f^{\prime}=1$ has infinitely many solutions.

Corresponding to Theorem A, C. C. Yang and X. H. Hua [14] proved the following result.

Theorem B. Let $f$ and $g$ be two nonconstant meromorphic functions, $n \geq 11$ be a positive integer. If $f^{n} f^{\prime}$ and $g^{n} g^{\prime}$ share $1 C M$, then either $f(z)=c_{1} e^{c z}$,

Received January 12, 2011; accepted July 24, 2012.

2010 Mathematics Subject Classification: 30D35.

Key words and phrases: Uniqueness, Meromorphic function, Nonlinear Differential Polynomials. 
$g(z)=c_{2} e^{-c z}$, where $c_{1}, c_{2}$ and $c$ are three constants satisfying $\left(c_{1} c_{2}\right)^{n+1} c^{2}=-1$ or $f \equiv t g$ for a constant $t$ such that $t^{n+1}=1$.

In 2000, M. L. Fang [4] proved the following result:

Theorem C. Let $f$ be a transcendental meromorphic function, and let $n$ be a positive integer. Then $f^{n} f^{\prime}-z=0$ has infinitely many solutions.

Corresponding to Theorem C, the following result was proved by M. L. Fang and H. L. Qiu [5].

Theorem D. Let $f$ and $g$ be two nonconstant meromorphic functions, and let $n \geq 11$ be a positive integer. If $f^{n} f^{\prime}-z$ and $g^{n} g^{\prime}-z$ share $0 C M$, then either $f(\bar{z})=c_{1} e^{c z^{2}}, g(z)=c_{2} e^{-c z^{2}}$, where $c_{1}, c_{2}$ and $c$ are three nonzero complex numbers satisfying $4\left(c_{1} c_{2}\right)^{n+1} c^{2}=-1$ or $f=t g$ for a complex number $t$ such that $t^{n+1}=1$.

In 2003, W. Bergweiler and X.C. Pang [3] proved the following result:

Theorem E. Let $f$ be a transcendental meromorphic function, and let $R \not \equiv 0$ be a rational function. If all zeros and poles of $f$ are multiple, except possibly finitely many, then $f^{\prime}-R=0$ has infinitely many solutions.

The question arises:

Question 1. Is there exist a uniqueness theorem corresponding to Theorem E, similar to Theorems B and D?

Recently X. M. Li and L. Gao [11] proved the following uniqueness theorems that deals with Question 1.

Theorem F. Let $f$ and $g$ be two transcendental meromorphic functions, let $n \geq 11$ be a positive integer, and let $P \not \equiv 0$ be a polynomial with its degree $\gamma_{P} \leq 11$. If $f^{n} f^{\prime}-P$ and $g^{n} g^{\prime}-P$ share $0 C M$, then either $f=t g$ for a complex number $t$ satisfying $t^{n+1}=1$, or $f=c_{1} e^{c Q}$ and $g=c_{2} e^{-c Q}$, where $c_{1}, c_{2}$ and $c$ are three nonzero complex numbers satisfying $\left(c_{1} c_{2}\right)^{n+1} c^{2}=-1, Q$ is a polynomial satisfying $Q=\int_{0}^{z} P(\eta) d \eta$.

Theorem G. Let $f$ and $g$ be two transcendental meromorphic functions, let $n \geq 15$ be a positive integer, and let $P \not \equiv 0$ be a polynomial. If $\left(f^{n}(f-1)\right)^{\prime}-P$ and $\left(g^{n}(g-1)\right)^{\prime}-P$ share $0 C M$ and $\Theta(\infty, f)>2 / n$, then $f=g$.

However questions arise in one's mind which are the motive of the author.

Question 2. Is it possible to obtain the similar result as in Theorems $\mathrm{F}$ and $\mathrm{G}$ if the sharing value is relaxed from CM to IM ?

Question 3. What happened if one consider kth derivative instead of first in Theorems $\mathrm{F}$ and G?

Considering $k$ th derivative, recently the present author [12] proved the following theorem. 
Theorem H. Let $f$ and $g$ be two transcendental meromorphic functions, and let $n(\geq 1), k(\geq 1)$ and $m(\geq 1)$ be three integers. Let $\left[f^{n}(f-1)^{m}\right]^{(k)}$ and $\left[g^{n}(g-1)^{m}\right]^{(k)}$ share the value $1 \mathrm{IM}$. Then one of the following holds:

(i) when $m=1, n>9 k+20$ and $\Theta(\infty, f)>\frac{2}{n}$, then either $\left[f^{n}(f-1)^{m}\right]^{(k)}\left[g^{n}(g-\right.$ 1) $\left.{ }^{m}\right]^{(k)} \equiv 1$ or $f \equiv g$

(ii) when $m \geq 2$ and $n>9 k+4 m+16$, then either $\left[f^{n}(f-1)^{m}\right]^{(k)}\left[g^{n}(g-1)^{m}\right]^{(k)} \equiv 1$ or $f \equiv g$ or $f$ and $g$ satisfy the algebraic equation $R(f, g)=0$, where

$$
R(x, y)=x^{n}(x-1)^{m}-y^{n}(y-1)^{m} .
$$

The possibility $\left[f^{n}(f-1)^{m}\right]^{(k)}\left[g^{n}(g-1)^{m}\right]^{(k)} \equiv 1$ does not arise for $k=1$.

So it is natural to ask the question:

Question 4. What can be said if we replace the sharing value 1 in the above theorem by a nonzero polynomial?

In the paper, we shall try to find out the possible solution of the above three questions. We will prove two theorems of which second one will not only improve and generalize Theorem $\mathrm{H}$ and at the same time provide a supplementary and generalize result of Theorem G. Our first theorem will supplement and generalize Theorem F. The following theorems are the main results of the paper.

Theorem 1. Let $f$ and $g$ be two transcendental meromorphic functions, let $n, k$ be two positive integers such that $n \geq 9 k+15$, and let $P \not \equiv 0$ be a polynomial with its degree $\gamma_{P} \leq n-1$. Let $\left(f^{n}\right)^{(k)}-P$ and $\left(g^{n}\right)^{(k)}-P$ share 0 IM. Then

(i) if $k=1$, either $f=t g$ for a complex number $t$ satisfying $t^{n}=1$ or $f=c_{1} e^{c Q}$ and $g=c_{2} e^{-c Q}$, where $c_{1}, c_{2}$ and $c$ are three nonzero complex numbers satisfying $\left(c_{1} c_{2}\right)^{n} c^{2}=-1, Q$ is a polynomial satisfying $Q=\int_{0}^{z} P(\eta) d \eta$;

(ii) if $k \geq 2$, either $\left(f^{n}\right)^{(k)}\left(g^{n}\right)^{(k)}=P^{2}$ or $f=t g$ for a complex number $t$ satisfying $t^{n}=1$.

Theorem 2. Let $f$ and $g$ be two transcendental meromorphic functions, let $n, m$, $k$ be three positive integers, and let $P \not \equiv 0$ be a polynomial. If $\left(f^{n}(f-1)^{m}\right)^{(k)}-P$ and $\left(g^{n}(g-1)^{m}\right)^{(k)}-P$ share 0 IM, then each of the following holds:

(i) when $m=1, n>9 k+20$ and $\Theta(\infty, f)+\Theta(\infty, g)>4 / n$, then either $\left(f^{n}(f-\right.$ $\left.1)^{m}\right)^{(k)}\left(g^{n}(g-1)^{m}\right)^{(k)}=P^{2}$ or $f=g$;

(ii) when $m \geq 2$ and $n>9 k+4 m+16$, then either $\left(f^{n}(f-1)^{m}\right)^{(k)}\left(g^{n}(g-1)^{m}\right)^{(k)}=$ $P^{2}$ or $f=g$ or $f$ and $g$ satisfy the algebraic equation $R(f, g)=0$, where

$$
R(x, y)=x^{n}(x-1)^{m}-y^{n}(y-1)^{m} .
$$

The possibility $\left(f^{n}(f-1)^{m}\right)^{(k)}\left(g^{n}(g-1)^{m}\right)^{(k)}=P^{2}$ does not arise for $k=1$.

We now explain some definitions and notations which are used in the paper.

Definition 1([9]). For $a \in \mathbb{C} \cup\{\infty\}$ we denote by $N(r, a ; f \mid=1)$ the counting functions of simple $a$-points of $f$. For a positive integer $p$ we denote by $N(r, a ; f \mid \leq$ 
$p$ ) the counting function of those $a$-points of $f$ (counted with proper multiplicities) whose multiplicities are not greater than $p$. By $\bar{N}(r, a ; f \mid \leq p)$ we denote the corresponding reduced counting function.

Analogously we can define $N(r, a ; f \mid \geq p)$ and $\bar{N}(r, a ; f \mid \geq p)$.

Definition 2([8]). Let $p$ be a positive integer or infinity. We denote by $N_{p}(r, a ; f)$ the counting function of $a$-points of $f$, where an $a$-point of multiplicity $m$ is counted $m$ times if $m \leq p$ and $p$ times if $m>p$. Then

$$
N_{p}(r, a ; f)=\bar{N}(r, a ; f)+\bar{N}(r, a ; f \mid \geq 2)+\ldots+\bar{N}(r, a ; f \mid \geq p) .
$$

Clearly $N_{1}(r, a ; f)=\bar{N}(r, a ; f)$.

Definition 3. For $a \in \mathbb{C} \cup\{\infty\}$ we define

$$
\delta_{p}(a, f)=1-\limsup _{r \longrightarrow \infty} \frac{N_{p}(r, a ; f)}{T(r, f)}
$$

and

$$
\Theta(a, f)=1-\limsup _{r \longrightarrow \infty} \frac{\bar{N}(r, a ; f)}{T(r, f)},
$$

where $p$ is an arbitrary nonnegative integer.

Remark 1. From the above definition it is clear that

$$
0 \leq \delta_{p}(a, f) \leq \delta_{p-1}(a, f) \leq \delta_{1}(a, f) \leq \Theta(a, f) \leq 1 .
$$

Definition 4([1,2]). Let $f$ and $g$ be two nonconstant meromorphic functions such that $f$ and $g$ share the value 1 IM. Let $z_{0}$ be a 1-point of $f$ with multiplicity $p$ and also a 1-point of $g$ with multiplicity $q$. We denote by $\bar{N}_{L}(r, 1 ; f)$ the reduced counting function of those 1-points of $f$ and $g$, where $p>q$, by $N_{E}^{1)}(r, 1 ; f)$ the counting function of those 1-points of $f$ and $g$, where $p=q=1$, by $\bar{N}_{E}^{(2}(r, 1 ; f)$ the reduced counting function of those 1-points of $f$ and $g$, where $p=q \geq 2$. Similarly we can define $\bar{N}_{L}(r, 1 ; g), N_{E}^{1)}(r, 1 ; g)$ and $\bar{N}_{E}^{(2}(r, 1 ; g)$.

\section{Lemmas}

In this section we present some lemmas which will be needed in the sequel.

Lemma 1([13]). Let $f$ be a transcendental meromorphic function, and let $P_{n}(f)$ be a differential polynomial in $f$ of the form

$$
P_{n}(f)=a_{n} f^{n}(z)+a_{n-1} f^{n-1}(z)+\ldots+a_{1} f(z)+a_{0},
$$

where $a_{n}(\neq 0), a_{n-1}, \ldots, a_{1}, a_{0}$ are complex numbers. Then

$$
T\left(r, P_{n}(f)\right)=n T(r, f)+O(1) .
$$


Lemma 2([17]). Let $f$ and $g$ be two nonconstant meromorphic functions, and let $p, k$ be two positive integers. Then

$$
N_{p}\left(r, 0 ; f^{(k)}\right) \leq N_{p+k}(r, 0 ; f)+k \bar{N}(r, \infty ; f)+S(r, f) .
$$

Lemma $3([7,15])$. Let $f$ be a transcendental meromorphic function, and let $a_{1}(z)$, $a_{2}(z)$ be two distinct meromorphic functions such that $T\left(r, a_{i}(z)\right)=S(r, f), i=1$, 2. Then

$$
T(r, f) \leq \bar{N}(r, \infty ; f)+\bar{N}\left(r, a_{1} ; f\right)+\bar{N}\left(r, a_{2} ; f\right)+S(r, f) .
$$

Lemma 4([7]). Let $f$ be a nonconstant meromorphic function, $k$ be a positive integer, and let $c$ be a nonzero finite complex number. Then

$$
\begin{aligned}
T(r, f) & \leq \bar{N}(r, \infty ; f)+N(r, 0 ; f)+N\left(r, c ; f^{(k)}\right)-N\left(r, 0 ; f^{(k+1)}\right)+S(r, f) \\
& \leq \bar{N}(r, \infty ; f)+N_{k+1}(r, 0 ; f)+\bar{N}\left(r, c ; f^{(k)}\right)-N_{0}\left(r, 0 ; f^{(k+1)}\right)+S(r, f),
\end{aligned}
$$

where $N_{0}\left(r, 0 ; f^{(k+1)}\right)$ denotes the counting function which only counts those points such that $f^{(k+1)}=0$ but $f\left(f^{(k)}-c\right) \neq 0$.

Lemma 5. Let $f$ and $g$ be two transcendental meromorphic functions such that $f^{(k)}-P$ and $g^{(k)}-P$ share $0 I M$, where $k$ is a positive integer, $P \not \equiv 0$ is a polynomial. If

$$
\begin{aligned}
\Delta_{1}= & (2 k+4) \Theta(\infty, f)+(2 k+3) \Theta(\infty, g)+\Theta(0, f)+\Theta(0, g) \\
& +3 \delta_{k+1}(0, f)+2 \delta_{k+1}(0, g)>4 k+13
\end{aligned}
$$

and

$$
\begin{aligned}
\Delta_{2}= & (2 k+4) \Theta(\infty, g)+(2 k+3) \Theta(\infty, f)+\Theta(0, g)+\Theta(0, f) \\
& +3 \delta_{k+1}(0, g)+2 \delta_{k+1}(0, f)>4 k+13,
\end{aligned}
$$

then either $f^{(k)} g^{(k)}=P^{2}$ or $f=g$.

Proof. Since $f$ and $g$ are two transcendental meromorphic functions, $f^{(k)}$ and $g^{(k)}$ are also two transcendental meromorphic functions. Let

$$
F=\frac{f^{(k)}}{P}, \quad G=\frac{g^{(k)}}{P}
$$

and let

$$
H=\left(\frac{F^{\prime \prime}}{F^{\prime}}-\frac{2 F^{\prime}}{F-1}\right)-\left(\frac{G^{\prime \prime}}{G^{\prime}}-\frac{2 G^{\prime}}{G-1}\right)
$$


Let $z_{0} \notin\{z: P(z)=0\}$ be a common simple zero of $f^{(k)}-P$ and $g^{(k)}-P$. Then $z_{0}$ is a common simple zero of $F-1$ and $G-1$. Substituting their Taylor series at $z_{0}$ into (2.3), we see that $z_{0}$ is a zero of $H$. Thus we have

$$
\begin{aligned}
N_{E}^{1)}(r, 1 ; F) & \leq N(r, 0 ; H) \leq T(r, H)+O(1) \\
& \leq N(r, \infty ; H)+S(r, F)+S(r, G) .
\end{aligned}
$$

Let $z_{1} \notin\{z: P(z)=0\}$ be a pole of $H$. Then from (2.3) we can see that $H$ have poles only at the zeros of $F^{\prime}$ and $G^{\prime}$, 1-points of $F$ whose multiplicities are not equal to the multiplicities of the corresponding 1-points of $G$, and poles of $f$ and $g$. Hence we have

(2.5) $N(r, \infty ; H) \leq \bar{N}(r, \infty ; f)+\bar{N}(r, \infty ; g)+\bar{N}(r, 0 ; f)+\bar{N}(r, 0 ; g)+\bar{N}_{L}(r, 1 ; F)$

$$
+\bar{N}_{L}(r, 1 ; G)+N_{0}\left(r, 0 ; F^{\prime}\right)+N_{0}\left(r, 0 ; G^{\prime}\right)+O(\log r),
$$

where $N_{0}\left(r, 0 ; F^{\prime}\right)$ denotes the counting function of those zeros of $F^{\prime}$ which are not the zeros of $f(F-1), N_{0}\left(r, 0 ; G^{\prime}\right)$ is similarly defined. Since $f$ is a transcendental meromorphic functions we have

$$
T(r, P)=o\{T(r, f)\} .
$$

By Lemma 4, we have

$$
\begin{aligned}
T(r, f) \leq & \bar{N}(r, \infty ; f)+N_{k+1}(r, 0 ; f)+\bar{N}(r, 1 ; F) \\
& -N_{0}\left(r, 0 ; F^{\prime}\right)+S(r, f) .
\end{aligned}
$$

Similarly

$$
\begin{aligned}
T(r, g) \leq & \bar{N}(r, \infty ; g)+N_{k+1}(r, 0 ; g)+\bar{N}(r, 1 ; G) \\
& -N_{0}\left(r, 0 ; G^{\prime}\right)+S(r, g) .
\end{aligned}
$$

Since $f^{(k)}-P$ and $g^{(k)}-P$ share 0 IM, therefore using (2.4) and (2.5) we obtain

$$
\begin{aligned}
\bar{N}(r, 1 ; F)+\bar{N}(r, 1 ; G)= & 2 N_{E}^{1)}(r, 1 ; F)+2 \bar{N}_{L}(r, 1 ; F)+2 \bar{N}_{L}(r, 1 ; G) \\
& +2 \bar{N}_{E}^{(2}(r, 1 ; F) \\
\leq & N_{E}^{1)}(r, 1 ; F)+\bar{N}(r, \infty ; f)+\bar{N}(r, \infty ; g) \\
& +\bar{N}(r, 0 ; f)+\bar{N}(r, 0 ; g)+3 \bar{N}_{L}(r, 1 ; F) \\
& +3 \bar{N}_{L}(r, 1 ; G)+N_{0}\left(r, 0 ; F^{\prime}\right)+N_{0}\left(r, 0 ; G^{\prime}\right) \\
& +2 \bar{N}_{E}^{(2}(r, 1 ; F)+S(r, f)+S(r, g)
\end{aligned}
$$

Obviously

$$
\begin{aligned}
& N_{E}^{1)}(r, 1 ; F)+2 \bar{N}_{E}^{(2}(r, 1 ; F)+\bar{N}_{L}(r, 1 ; F)+2 \bar{N}_{L}(r, 1 ; G) \\
& \quad \leq N(r, 1 ; G)+S(r, f)+S(r, g) \\
& \quad \leq T(r, G)+S(r, f)+S(r, g) \\
& \quad \leq T(r, g)+k \bar{N}(r, \infty ; g)+S(r, f)+S(r, g) .
\end{aligned}
$$


Also by Lemma 3 we have

$$
\begin{aligned}
\bar{N}_{L}(r, 1 ; F) & \leq N(r, 1 ; F)-\bar{N}(r, 1 ; F) \\
& \leq N\left(r, \infty ; \frac{F}{F^{\prime}}\right) \\
& \leq N\left(r, \infty ; \frac{F^{\prime}}{F}\right)+S(r, f) \\
& \leq \bar{N}(r, 0 ; F)+\bar{N}(r, \infty ; f)+S(r, f) \\
& \leq N_{k+1}(r, 0 ; f)+(k+1) \bar{N}(r, \infty ; f)+S(r, f) .
\end{aligned}
$$

Similarly,

$$
\bar{N}_{L}(r, 1 ; G) \leq N_{k+1}(r, 0 ; g)+(k+1) \bar{N}(r, \infty ; g)+S(r, g) .
$$

From (2.7) - (2.12), we obtain

(2.13) $T(r, f) \leq(2 k+4) \bar{N}(r, \infty ; f)+(2 k+3) \bar{N}(r, \infty ; g)+\bar{N}(r, 0 ; f)+\bar{N}(r, 0 ; g)$ $+3 N_{k+1}(r, 0 ; f)+2 N_{k+1}(r, 0 ; g)+S(r, f)+S(r, g)$.

Similarly,

$$
\begin{aligned}
T(r, g) \leq & (2 k+4) \bar{N}(r, \infty ; g)+(2 k+3) \bar{N}(r, \infty ; f)+\bar{N}(r, 0 ; g)+\bar{N}(r, 0 ; f) \\
& +3 N_{k+1}(r, 0 ; g)+2 N_{k+1}(r, 0 ; f)+S(r, f)+S(r, g) .
\end{aligned}
$$

Suppose that there exists a subset $I \subseteq R^{+}$satisfying $m e s I=\infty$ such that $T(r, g) \leq$ $T(r, f), r \in I$. Hence from (2.13) we have

$$
\begin{aligned}
\Delta_{1}= & (2 k+4) \Theta(\infty, f)+(2 k+3) \Theta(\infty, g)+\Theta(0, f)+\Theta(0, g) \\
& +3 \delta_{k+1}(0, f)+2 \delta_{k+1}(0, g) \leq 4 k+13,
\end{aligned}
$$

contradicting (2.1). Similarly if there exists a subset $I \subseteq R^{+}$satisfying mesI $=\infty$ such that $T(r, f) \leq T(r, g), r \in I$, from (2.14) we can obtain

$$
\begin{aligned}
\Delta_{2}= & (2 k+4) \Theta(\infty, g)+(2 k+3) \Theta(\infty, f)+\Theta(0, g)+\Theta(0, f) \\
& +3 \delta_{k+1}(0, g)+2 \delta_{k+1}(0, f) \leq 4 k+13,
\end{aligned}
$$

contradicting (2.2). We now assume that $H=0$. That is

$$
\left(\frac{F^{\prime \prime}}{F^{\prime}}-\frac{2 F^{\prime}}{F-1}\right)-\left(\frac{G^{\prime \prime}}{G^{\prime}}-\frac{2 G^{\prime}}{G-1}\right)=0 .
$$

Integrating both sides of the above equality twice we get

$$
\frac{1}{F-1}=\frac{A}{G-1}+B
$$

where $A(\neq 0)$ and $B$ are finite complex constants. We now discuss the following three cases. 
Case 1. Let $B \neq 0$ and $A=B$. If $B=-1$, we obtain from (2.15) $F G=1$, i.e., $f^{(k)} g^{(k)}=P^{2}$. If $B \neq-1$, from (2.15) we get

$$
\frac{1}{F}=\frac{B G}{(1+B) G-1} \text { and } G=\frac{-1}{b\left(F-\frac{1+B}{B}\right)} .
$$

So by Lemma 2 we obtain

$$
\begin{aligned}
\bar{N}\left(r, \frac{1}{1+B} ; G\right) \leq & \bar{N}(r, 0 ; F) \leq N_{k+1}(r, 0 ; f)+k \bar{N}(r, \infty ; f) \\
& +O(\log r)+S(r, f)
\end{aligned}
$$

and

$$
\bar{N}\left(r, \frac{1+B}{B} ; F\right) \leq \bar{N}(r, \infty ; g)+O(\log r) .
$$

Using Lemma 4, (2.16) and (2.17) we obtain

$$
\begin{aligned}
T(r, g) \leq & N_{k+1}(r, 0 ; g)+\bar{N}\left(r, \frac{1}{1+B} ; G\right)+\bar{N}(r, \infty ; g) \\
& -N_{0}\left(r, 0 ; G^{\prime}\right)+S(r, g) \\
\leq & N_{k+1}(r, 0 ; g)+N_{k+1}(r, 0 ; f)+k \bar{N}(r, \infty ; f) \\
& +\bar{N}(r, \infty ; g)+S(r, f)+S(r, g)
\end{aligned}
$$

and

$$
\begin{aligned}
T(r, f) \leq & N_{k+1}(r, 0 ; f)+\bar{N}\left(r, \frac{1+B}{B} ; F\right)+\bar{N}(r, \infty ; f) \\
& -N_{0}\left(r, 0 ; F^{\prime}\right)+S(r, f) \\
\leq & N_{k+1}(r, 0 ; f)+\bar{N}(r, \infty ; f)+\bar{N}(r, \infty ; g)+S(r, f) .
\end{aligned}
$$

Suppose that there exists a subset $I \subseteq R^{+}$satisfying mesI $=\infty$ such that $T(r, f) \leq$ $T(r, g), r \in I$. So from (2.18) we obtain

$$
k \Theta(\infty, f)+\Theta(\infty, g)+\delta_{k+1}(0, f)+\delta_{k+1}(0, g) \leq k+2,
$$

which by (2.1) gives

$$
\begin{aligned}
(k+4) \Theta(\infty, f)+ & (2 k+2) \Theta(\infty, g)+\Theta(0, f)+\Theta(0, g) \\
+ & +2 \delta_{k+1}(0, f)+\delta_{k+1}(0, g)>3 k+11,
\end{aligned}
$$

a contradiction together with Remark 1. If there exists a subset $I \subseteq R^{+}$satisfying mesI $=\infty$ such that $T(r, g) \leq T(r, f), r \in I$, by the same argument we obtain a contradiction from (2.1) and (2.19). 
Case 2. Let $B \neq 0$ and $A \neq B$. If $B=-1$, from (2.15) we obtain $F=\frac{A}{-(G-(a+1))}$. If $B \neq-1$, from (2.15) we obtain $F-\frac{1+B}{B}=\frac{-A}{B^{2}\left(G+\frac{A-B}{B}\right)}$. Using the same argument as in case 1 we obtain a contradiction in both the cases.

Case 3. Let $B=0$. Then from (2.15) we get

$$
g=A f+(1-A) P_{1},
$$

where $P_{1}$ is a polynomial of degree $\gamma_{P_{1}} \geq k$. If $A \neq 1$, by Lemma 3 and (2.20) we get

$$
\begin{aligned}
T(r, g) & \leq \bar{N}(r, 0 ; g)+\bar{N}(r, \infty ; g)+\bar{N}\left(r,(1-A) P_{1} ; g\right)+S(r, g) \\
& \leq \bar{N}(r, 0 ; g)+\bar{N}(r, \infty ; g)+\bar{N}(r, 0 ; f)+S(r, g) .
\end{aligned}
$$

Since $f$ and $g$ are transcendental meromorphic function, from (2.20) we have

$$
T(r, f)=T(r, g)+O(\log r) .
$$

So from (2.21), we obtain

$$
\Theta(0, f)+\Theta(0, g)+\Theta(\infty, g) \leq 2,
$$

which gives by $(2.1)$

$$
(2 k+4) \Theta(\infty, f)+(2 k+2) \Theta(\infty, g)+3 \delta_{k+1}(0, f)+2 \delta_{k+1}(0, g)>4 k+11,
$$

a contradiction together with Remark 1 . Thus $A=1$ and so $f=g$. This proves the lemma.

Lemma 6. Let $f$ and $g$ be two nonconstant meromorphic functions such that

$$
\Theta(\infty, f)+\Theta(\infty, g)>\frac{4}{n},
$$

where $n(\geq 3)$ is an integer. Then

$$
f^{n}(a f+b) \equiv g^{n}(a g+b)
$$

implies $f \equiv g$, where $a, b$ are two nonzero constants.

Proof. We omit the proof since it can be carried out in the line of Lemma 6 [10].

Lemma $7([\mathbf{1 1}])$. Let $f$ and $g$ be two transcendental meromorphic functions, let $n \geq 2$ be a positive integer, and let $P$ be a nonconstant polynomial with its degree $\gamma_{P} \leq n$. If $f^{n} f^{\prime} g^{n} g^{\prime}=P^{2}$, then $f$ and $g$ are expressed as $f=c_{1} e^{c Q}$ and $g=$ $c_{2} e^{-c Q}$ respectively, where $c_{1}, c_{2}$ and $c$ are three nonzero complex numbers satisfying $\left(c_{1} c_{2}\right)^{n+1} c^{2}=-1, Q$ is a polynomial satisfying $Q=\int_{0}^{z} P(\eta) d \eta$. 
Lemma 8. Let $f$ and $g$ be two transcendental meromorphic functions, let $n, m$ be two positive integers and let $P$ be a nonconstant polynomial. If $m=1, n \geq 6$ or if $m \geq 2, n \geq m+3$, then

$$
\left(f^{n}(f-1)^{m}\right)^{\prime}\left(g^{n}(g-1)^{m}\right)^{\prime} \neq P^{2} .
$$

Proof. If possible, let

$$
\left(f^{n}(f-1)^{m}\right)^{\prime}\left(g^{n}(g-1)^{m}\right)^{\prime}=P^{2} .
$$

We discuss the following two cases.

Case 1. Let $m \geq 2$. Then from (2.22) we obtain

$$
f^{n-1}(f-1)^{m-1}(c f-d) f^{\prime} g^{n-1}(g-1)^{m-1}(c g-d) g^{\prime}=P^{2},
$$

where $c=n+m$ and $d=n$.

Let $z_{0} \notin\{z: P(z)=0\}$ be a 1-point of $f$ with multiplicity $p_{0}(\geq 1)$. Then from (2.23) it follows that $z_{0}$ is a pole of $g$. Suppose that $z_{0}$ is a pole of $g$ of order $q_{0}(\geq 1)$. Then we have $m p_{0}-1=(n+m) q_{0}+1$, i.e., $m p_{0}=(n+m) q_{0}+2 \geq n+m+2$, and so

$$
p_{0} \geq \frac{n+m+2}{m} .
$$

Let $z_{1} \notin\{z: P(z)=0\}$ be a zero of $c f-d$ with multiplicity $p_{1}(\geq 1)$. Then from (2.23) it follows that $z_{1}$ is a pole of $g$. Suppose that $z_{1}$ is a pole of $g$ of order $q_{1}(\geq 1)$. Then we have $2 p_{1}-1=(n+m) q_{1}+1$, and so

$$
p_{1} \geq \frac{n+m+2}{2} .
$$

Let $z_{2} \notin\{z: P(z)=0\}$ be a zero of $f$ with multiplicity $p_{2}(\geq 1)$. Then it follows from (2.23) that $z_{2}$ is a pole of $g$. Suppose that $z_{2}$ is a pole of $g$ of order $q_{2}(\geq 1)$. Then we have

$$
n p_{2}-1=(n+m) q_{2}+1 .
$$

From (2.24) we get $m q_{2}+2=n\left(p_{2}-q_{2}\right) \geq n$, i.e., $q_{2} \geq \frac{n-2}{m}$. Thus from (2.24) we obtain $n p_{2}=(n+m) q_{2}+2 \geq \frac{(n+m)(n-2)}{m}+2$, and so

$$
p_{2} \geq \frac{n+m-2}{m} \text {. }
$$

Let $z_{3} \notin\{z: P(z)=0\}$ be a pole of $f$. Then it follows from (2.23) that $z_{3}$ is a zero 
of $g(g-1)(c g-d)$ or a zero of $g^{\prime}$. So we have

$$
\begin{aligned}
\bar{N}(r, \infty ; f) \leq & \bar{N}(r, 0 ; g)+\bar{N}(r, 1 ; g)+\bar{N}\left(r, \frac{d}{c} ; g\right)+\bar{N}_{0}\left(r, 0 ; g^{\prime}\right) \\
& +S(r, f)+S(r, g) \\
\leq & \left(\frac{m+2}{n+m+2}+\frac{m}{n+m-2}\right) T(r, g)+\bar{N}_{0}\left(r, 0 ; g^{\prime}\right) \\
& +S(r, f)+S(r, g),
\end{aligned}
$$

where $\bar{N}_{0}\left(r, 0 ; g^{\prime}\right)$ denotes the reduced counting function of those zeros of $g^{\prime}$ which are not the zeros of $g(g-1)(c g-d)$.

By the second fundamental theorem of Nevanlinna we get

$$
\begin{aligned}
2 T(r, f) \leq & \bar{N}(r, 0 ; f)+\bar{N}(r, 1 ; f)+\bar{N}\left(r, \frac{d}{c} ; f\right)+\bar{N}(r, \infty ; f) \\
& -\bar{N}_{0}\left(r, 0 ; f^{\prime}\right)+S(r, f) \\
\leq & \left(\frac{m+2}{n+m+2}+\frac{m}{n+m-2}\right)\{T(r, f)+T(r, g)\}-\bar{N}_{0}\left(r, 0 ; f^{\prime}\right) \\
& +\bar{N}_{0}\left(r, 0 ; g^{\prime}\right)+S(r, f)+S(r, g) .
\end{aligned}
$$

Similarly,

(2.26) $2 T(r, g) \leq\left(\frac{m+2}{n+m+2}+\frac{m}{n+m-2}\right)\{T(r, f)+T(r, g)\}+\bar{N}_{0}\left(r, 0 ; f^{\prime}\right)$

$$
-\bar{N}_{0}\left(r, 0 ; g^{\prime}\right)+S(r, f)+S(r, g) \text {. }
$$

Adding (2.25) and (2.26) we obtain

$$
\left(1-\frac{m+2}{n+m+2}-\frac{m}{n+m-2}\right)\{T(r, f)+T(r, g)\} \leq S(r, f)+S(r, g),
$$

contradicting the fact that $n \geq m+3$.

Case 2. Let $m=1$. Then from (2.22) we obtain

$$
f^{n-1}(a f-b) f^{\prime} g^{n-1}(a g-b) g^{\prime}=P^{2},
$$

where $a=n+1$ and $b=n$.

Let $z_{4} \notin\{z: P(z)=0\}$ be a pole of $f$. Then it follows from (2.27) that $z_{4}$ is a zero of $g(a g-b)$ or a zero of $g^{\prime}$. Then proceeding in a manner similar to Case 1 we obtain

$$
\left(1-\frac{2}{n-1}-\frac{4}{n+3}\right)\{T(r, f)+T(r, g)\} \leq S(r, f)+S(r, g),
$$

which contradicts the fact that $n \geq 6$. This proves the lemma. 


\section{Proof of the Theorems}

Proof of Theorem 1. We consider $F_{1}=f^{n}$ and $G_{1}=g^{n}$. Then we see that $F_{1}^{(k)}-P$ and $G_{1}^{(k)}-P$ share the value 0 IM. Using Lemma 1 , we have

$$
\begin{aligned}
\Theta\left(0, F_{1}\right) & =1-\limsup _{r \rightarrow \infty} \frac{\bar{N}\left(r, 0 ; F_{1}\right)}{T\left(r, F_{1}\right)} \\
& =1-\limsup _{r \rightarrow \infty} \frac{\bar{N}(r, 0 ; f)}{n T(r, f)} \\
& \geq 1-\limsup _{r \rightarrow \infty} \frac{T(r, f)}{n T(r, f)} \\
& =\frac{n-1}{n} .
\end{aligned}
$$

Similarly,

$$
\begin{aligned}
\Theta\left(0, G_{1}\right) \geq \frac{n-1}{n} \\
\Theta\left(\infty, F_{1}\right)=1-\limsup _{r \rightarrow \infty} \frac{\bar{N}\left(r, \infty ; F_{1}\right)}{T\left(r, F_{1}\right)} \\
=1-\limsup _{r \rightarrow \infty} \frac{\bar{N}(r, \infty ; f)}{n T(r, f)} \\
\geq 1-\limsup _{r \rightarrow \infty} \frac{T(r, f)}{n T(r, f)} \\
=\frac{n-1}{n} .
\end{aligned}
$$

Similarly,

$$
\begin{aligned}
& \Theta\left(\infty, G_{1}\right) \geq \frac{n-1}{n} . \\
& \delta_{k+1}\left(0, F_{1}\right)=1-\limsup _{r \rightarrow \infty} \frac{N_{k+1}\left(r, 0 ; F_{1}\right)}{T\left(r, F_{1}\right)} \\
&=1-\limsup _{r \rightarrow \infty} \frac{N_{k+1}\left(r, 0 ; f^{n}\right)}{n T(r, f)} \\
& \geq 1-\limsup _{r \rightarrow \infty} \frac{(k+1) T(r, f)}{n T(r, f)} \\
&=\frac{n-k-1}{n} .
\end{aligned}
$$


Similarly,

$$
\delta_{k+1}\left(0, G_{1}\right) \geq \frac{n-k-1}{n} .
$$

Using (2.1), (2.2) and (3.1)-(3.6) we obtain

$$
\Delta_{1} \geq(4 k+14)-\frac{9 k+14}{n} \quad \text { and } \quad \Delta_{2} \geq(4 k+14)-\frac{9 k+14}{n} .
$$

Since $n \geq 9 k+15$, we get $\Delta_{1}>4 k+13$ and $\Delta_{2}>4 k+13$. So by Lemma 5 we obtain either $F_{1}^{(k)} G_{1}^{(k)}=P^{2}$ or $F_{1}=G_{1}$. Suppose that $F_{1}^{(k)} G_{1}^{(k)}=P^{2}$, i.e.,

$$
\left(f^{n}\right)^{(k)}\left(g^{n}\right)^{(k)}=P^{2} .
$$

If $k=1$, then from (3.7) we have $f^{n-1} f^{\prime} g^{n-1} g^{\prime}=P^{2} / n^{2}$. Applying Lemma 7 we obtain $f=c_{1} e^{c Q}$ and $g=c_{2} e^{-c Q}$, where $c_{1}, c_{2}$ and $c$ are three nonzero complex numbers satisfying $\left(c_{1} c_{2}\right)^{n} c^{2}=-1, Q$ is a polynomial satisfying $Q=\int_{0}^{z} P(\eta) d \eta$.

If $F_{1}=G_{1}$, then $f=t g$ for a complex number $t$ such that $t^{n}=1$. This completes the proof of Theorem 1 .

Proof of Theorem 2. Let $F_{2}=f^{n}(f-1)^{m}$ and $G_{2}=g^{n}(g-1)^{m}$. Then $F_{2}^{(k)}-P$ and $G_{2}^{(k)}-P$ share the value 0 IM. Using Lemma 1 , we obtain

$$
\begin{aligned}
\Theta\left(0, F_{2}\right) & =1-\limsup _{r \rightarrow \infty} \frac{\bar{N}\left(r, 0 ; F_{2}\right)}{T\left(r, F_{2}\right)} \\
& =1-\limsup _{r \rightarrow \infty} \frac{\bar{N}\left(r, 0 ; f^{n}(f-1)^{m}\right)}{(n+m) T(r, f)} \\
& \geq 1-\limsup _{r \rightarrow \infty} \frac{2 T(r, f)}{(n+m) T(r, f)} \\
& \geq \frac{n+m-2}{n+m} .
\end{aligned}
$$

Similarly,

$$
\begin{aligned}
\Theta\left(0, G_{2}\right) \geq \frac{n+m-2}{n+m} . \\
\Theta\left(\infty, F_{2}\right)=1-\limsup _{r \rightarrow \infty} \frac{\bar{N}\left(r, \infty ; F_{2}\right)}{T\left(r, F_{2}\right)} \\
=1-\limsup _{r \rightarrow \infty} \frac{\bar{N}(r, \infty ; f)}{(n+m) T(r, f)} \\
\geq 1-\limsup _{r \rightarrow \infty} \frac{T(r, f)}{(n+m) T(r, f)} \\
\geq \frac{n+m-1}{n+m} .
\end{aligned}
$$


Similarly,

$$
\begin{aligned}
& \Theta\left(\infty ; G_{2}\right) \geq \frac{n+m-1}{n+m} . \\
& \delta_{k+1}\left(0, F_{2}\right)=1-\limsup _{r \rightarrow \infty} \frac{N_{k+1}\left(r, 0 ; F_{2}\right)}{T\left(r, F_{2}\right)} \\
&=1-\limsup _{r \rightarrow \infty} \frac{N_{k+1}\left(r, 0 ; f^{n}(f-1)^{m}\right)}{(n+m) T(r, f)} \\
& \geq 1-\limsup _{r \rightarrow \infty} \frac{(k+m+1) T(r, f)}{(n+m) T(r, f)} \\
& \geq \frac{n-k-1}{n+m} .
\end{aligned}
$$

Similarly,

$$
\delta_{k+1}\left(0, G_{2}\right) \geq \frac{n-k-1}{n+m} .
$$

Using (2.1), (2.2) and (3.8)-(3.13) we obtain

$$
\Delta_{1} \geq(4 k+9)+\frac{5 n-9 k-16}{n+m} \quad \text { and } \quad \Delta_{2} \geq(4 k+9)+\frac{5 n-9 k-16}{n+m} .
$$

Since $n \geq 9 k+4 m+17$, we get $\Delta_{1}>4 k+13$ and $\Delta_{2}>4 k+13$. So by Lemma 5 , either $F_{2}^{(k)} G_{2}^{(k)}=P^{2}$ or $F_{2}=G_{2}$ holds. Suppose that $F_{2}^{(k)} G_{2}^{(k)}=P^{2}$. Then

$$
\left(f^{n}(f-1)^{m}\right)^{(k)}\left(g^{n}(g-1)^{m}\right)^{(k)}=P^{2} .
$$

Also by Lemma $8,(3.14)$ does not occur when $k=1$.

Next we suppose that $F_{2}=G_{2}$, i.e.,

$$
f^{n}(f-1)^{m}=g^{n}(g-1)^{m} .
$$

Let $m=1$. Then in view of Lemma 6 and (3.15) we obtain $f=g$.

Let $m \geq 2$. Then from (3.15) we obtain

$$
\begin{array}{r}
f^{n}\left[f^{m}+\ldots+(-1)^{i}{ }^{m} C_{i} f^{m-i}+\ldots+(-1)^{m}\right]=g^{n}\left[g^{m}\right. \\
\left.+\ldots+(-1)^{i}{ }^{m} C_{i} g^{m-i}+\ldots+(-1)^{m}\right] .
\end{array}
$$

Let $h=\frac{f}{g}$. If $h$ is a constant, then substituting $f=g h$ in (3.16) we obtain

$$
\begin{array}{r}
g^{n+m}\left(h^{n+m}-1\right)+\ldots+(-1)^{i}{ }^{m} C_{i} g^{n+m-i}\left(h^{n+m-i}-1\right) \\
+\ldots+(-1)^{m} g^{n}\left(h^{n}-1\right)=0
\end{array}
$$

which implies $h=1$. Hence $f=g$. 
If $h$ is not a constant, then from (3.15) we can say that $f$ and $g$ satisfy the algebraic equation $R(f, g)=0$, where

$$
R(x, y)=x^{n}(x-1)^{m}-y^{n}(y-1)^{m} .
$$

This completes the proof of Theorem 2.

\section{References}

[1] T. C. Alzahary and H. X. Yi, Weighted value sharing and a question of I. Lahiri, Complex Var. Theory Appl., 49(2004), 1063-1078.

[2] A. Banerjee, Meromorphic functions sharing one value, Int. J. Math. Math. Sci., 22(2005), 3587-3598.

[3] W. Bergweiler and X. C. Pang, On the derivative of meromorphic functions with multiple zeros, J. Math. Anal. Appl., 278(2003), 285-292.

[4] M. L. Fang, A note on a problem of Hayman, Analysis (Munich), 20(2000), 45-49.

[5] M. L. Fang and H. L. Qiu, Meromorphic functions that share fixed points, J. Math. Anal. Appl., 268(2002), 426-439.

[6] W. K. Hayman, Picard values of meromorphic Functions and their derivatives, Ann. of Math., 70(1959), 9-42.

[7] W. K. Hayman, Meromorphic Functions, The Clarendon Press, Oxford (1964).

[8] I. Lahiri, Weighted value sharing and uniqueness of meromorphic functions, Complex Var. Theory Appl., 46(2001), 241-253.

[9] I. Lahiri, Value distribution of certain differential polynomials, Int. J. Math. Math. Sc., 28(2001), 83-91.

[10] I. Lahiri, On a question of Hong Xun Yi, Arch Math. (Brno), 38(2002), 119-128.

[11] X. M. Li and L. Gao, Meromorphic functions sharing a nonzero polynomial CM, Bull. Korean Math. Soc., 47(2010), 319-339.

[12] P. Sahoo, Uniqueness of meromorphic functions when two differential polynomials share one value IM, Mat. Vesnik, 62(2010), 169-182.

[13] C. C. Yang, On deficiencies of differential polynomials II, Math. Z., 125(1972), 107112 .

[14] C. C. Yang and X. H. Hua, Uniqueness and value sharing of meromorphic functions, Ann. Acad. Sci. Fenn. Math., 22(1997), 395-406.

[15] L. Yang, Value Distribution Theory, Springer- Verlag, Berlin, 1993.

[16] H. X. Yi and C. C. Yang, Uniqueness Theory of Meromorphic Functions, Science Press, Beijing, 1995.

[17] Q. C. Zhang, Meromorphic function that shares one small function with its derivative, J. Inequal. Pure Appl. Math., 6(2005), Art. 116. 